\title{
Síndrome de Sturge-Weber: estudo ultrabiomicroscópico
}

\author{
Sturge-Weber syndrome:ultrasound biomicroscopic study
}

\author{
SebastiãoCronemberger ${ }^{1}$ \\ Nassim Calixto ${ }^{2}$ \\ Déborah Ag'uiar Mendonça Assunção ${ }^{3}$ \\ Eduardo Gutemberg' Milhomens ${ }^{4}$
}

\section{RESUMO}

Objetivo: Descrever os achados da biomicroscopia ultra-sônica(UBM) em pacientes com síndrome de Sturge-Weber associada ou não a glaucoma. Métodos: Onze olhos (dois sem glaucoma e nove com glaucoma) de oito pacientes portadores de síndrome de Sturge-Weber foram submetidos à biomicroscopia ultra-sônica objetivando-se estudar o seio camerular, a esclera, o corpo ciliar e a pars plana. Resultados: Identificou-se pela biomicroscopia ultra-sônica imagens compatíveis com a suspeita de vaso intraescleral dilatado em todos os olhos com ou sem glaucoma. Efusão supraciliar foi encontrada em oito $(88,9 \%)$ dos nove olhos com glaucoma, porém, em nenhum dos olhos sem glaucoma. Descolamento da pars plana foi diagnosticado em um olho com glaucoma e nos dois olhos sem glaucoma. Em oito $(88,9 \%)$ olhos com glaucoma e nos dois sem glaucoma, observou-se imagem arredondada apresentando baixa a média ecogenicidade no interior do corpo ciliar, sugestiva de vaso dilatado (possível angioma) do corpo ciliar. Conclusão: Na síndrome de Sturge-Weber, a biomicroscopia ultrasônica mostrou a presença de imagens sugestivas de vasos dilatados no interior da esclera e do corpo ciliar (possíveis angiomas) em olhos glaucomatosos e não glaucomatosos. Paradoxalmente, diagnosticou-se efusão supraciliar em oito $(88,9 \%)$ dos nove olhos com glaucoma. Estes achados combinados com o angioma coroidiano e o aumento da pressão venosa episcleral em percentual elevado de olhos com esta síndrome, podem contribuir para explicar a patogenia do glaucoma, bem como as complicações per e pós-operatórias que acompanham as cirurgias antiglaucomatosas nesses olhos.

Descritores: Síndrome de Sturge-Weber; Angiomatose; Glaucoma/ultra-sonografia; Microscopia; Esclera/ultra-sonografia; Corpo ciliar/ultra-sonografia

\section{INTRODUÇ̃̃̃O}

A síndrome de Sturge-Weber ou angiomatose encefalotrigeminal é uma doença neuro-oculocutânea congênita com malformações que acometem primordialmente o sistema vascular cefálico, sendo caracterizada por angiomas distribuídos principalmente nas $1^{a}$ e $2^{a}$ divisões do quinto nervo craniano (Figura 1).

Schirmer ${ }^{(1)}$ em 1860 parece ter sido o primeiro a relatar a associação de telangiectasias da face e do corpo com glaucoma infantil. Posteriormente, Sturge ${ }^{(2)}$ associou as alterações dermatológicas e oftalmológicas às manifestações neurológicas da doença e Weber ${ }^{(3)}$ documentou as alterações radiológicas cerebrais destes pacientes. Desde então, a doença vem sendo descrita como Doença de Sturge-Weber em homenagem aos autores que mais contribuíram para o seu conhecimento. 


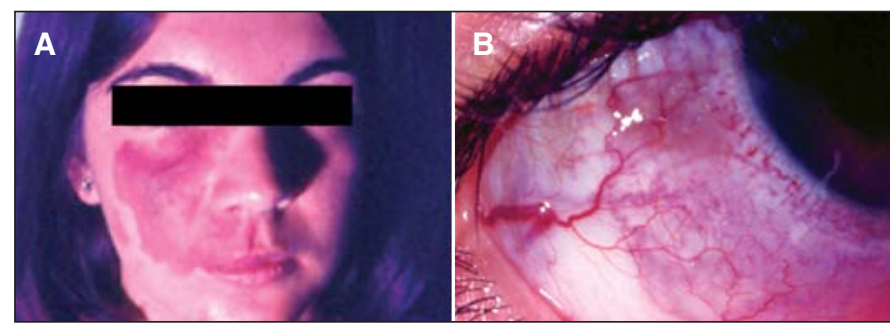

Figura 1 - Síndrome de Sturge-Weber: Foto ilustrativa mostrando: A) mancha de coloração vinhosa distribuída na $1^{\mathrm{a}}$ e $2^{\mathrm{a}}$ divisões do nervo trigêmeo; B) formação plexiforme vascular vinhosa perilímbica (angioma episcleral) no olho direito (maior aumento) (paciente 8)

A fisiopatologia do glaucoma secundário à síndrome de Sturge-Weber ainda não foi esclarecida ${ }^{(4-13)}$.

Jorgensen e Guthoff ${ }^{(11)}$ mediram a pressão venosa episcleral $(\mathrm{Pv})$ em pacientes portadores da síndrome de SturgeWeber (11 com glaucoma), tendo encontrado Pv elevada em todos os 11 pacientes glaucomatosos; em nove pacientes, encontraram pela ultra-sonografia um angioma de coróide difuso (1,5 a $4 \mathrm{~mm}$ de espessura da coróide).

Em nosso meio ${ }^{(12)}$, realizou-se estudo em portadores de síndrome de Sturge-Weber em que foi feita a medida da Pv em 38 olhos: 16 com glaucoma unilateral e 22 sem glaucoma numa casuística de 58 pacientes. A Pv foi medida $\mathrm{em}^{3} \mathrm{~cm}_{2} \mathrm{O}$ utilizando-se um instrumento acoplado à lâmpada de fenda Haag-Streit pelo qual uma câmara de pressão com uma fina membrana transparente foi aplicada ao vaso episcleral observando-se o colapso da veia sobre o qual a câmara estava apoiada. Multiplicandose o valor em $\mathrm{cm}^{3} \mathrm{H}_{2} \mathrm{O}$ por 0,734 obteve-se a Pv em mmHg. A Pv normal não varia com a idade. Encontraram Pv elevada (superior a $9 \mathrm{mmHg}$ ) em todos os 16 olhos glaucomatosos mas também em três olhos contralaterais normais (sem angioma episcleral e sem glaucoma). Foi encontrada Pv também elevada em dois olhos com angioma episcleral sem glaucoma. Não encontraram explicação para o aumento da $\mathrm{Pv}$ nos dois olhos com angioma episcleral sem glaucoma e nos três olhos normais (sem angioma episcleral e sem glaucoma). Esses mesmos autores ${ }^{(12)}$, utilizando uma sonda de $8 \mathrm{MHz}$ do Eco-oftalmógrafo Kretz modelo 7200 MA, mediram também a espessura da coróide de olhos com síndrome de Sturge-Weber encontrando grande aumento da mesma em todos os olhos com angioma de coróide reconhecido pela oftalmoscopia (Figura 2).

A biomicroscopia ultra-sônica (UBM) é um método de imagem que utiliza ultra-som de alta frequêencia $(50 \mathrm{MHz})$ para o estudo do segmento anterior do olho ensejando o esclarecimento fisiopatológico de muitas doenças que acometem essa região ${ }^{(14)}$.

Kranemann et al ${ }^{(15)}$, utilizando a UBM, estudaram um caso de glaucoma associado à síndrome de Sturge-Weber. Os autores relataram ter encontrado efusão supraciliar em $360^{\circ}$, vasos superficiais e intra-esclerais dilatados e seio camerular aberto, porém estreito, com o estreitamento provavelmente causado por alguma rotação do corpo ciliar secundária à efusão supraciliar. Estas alterações, segundo esses autores, sustentariam a hipótese de o aumento da pressão venosa episcleral (não

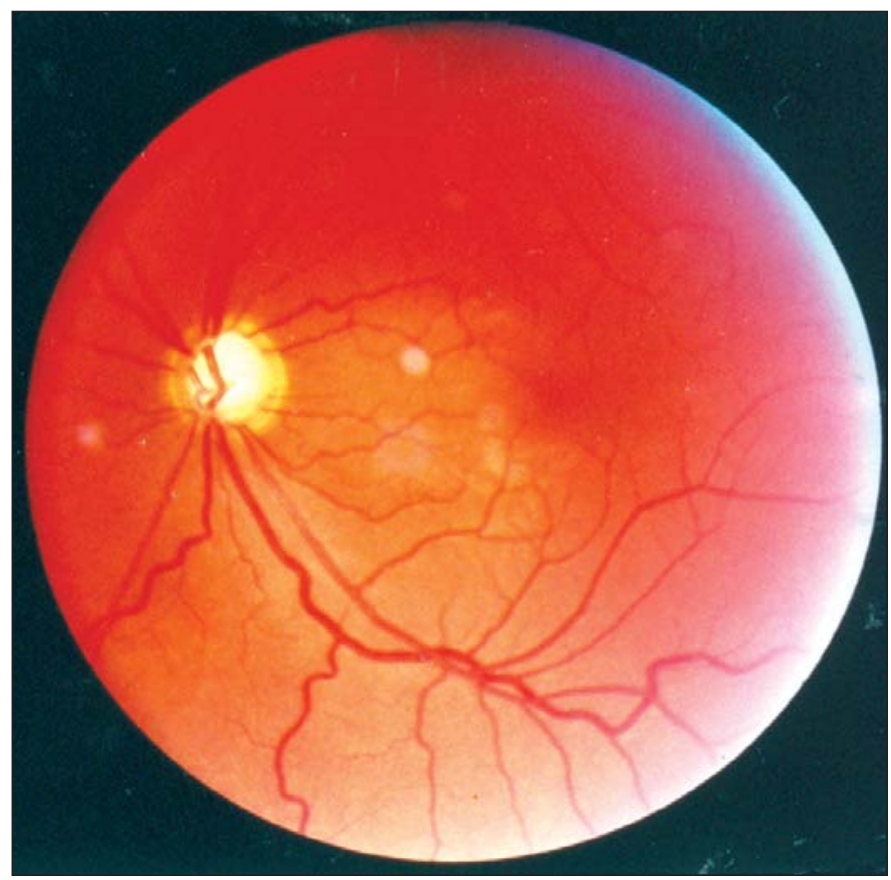

Figura 2 - Retinografia ilustrativa mostrando uma coloração vinhosa e difusa da coróide, compatível com a suspeita diagnóstica de angioma da coróide no olho esquerdo (paciente portador de síndrome de SturgeWeber e glaucoma secundário) (paciente 8)

medida por eles) ser a causa da elevação da pressão intraocular. Este foi o único relato da literatura compulsada empregando a UBM no estudo da síndrome de Sturge-Weber.

Assim sendo, o presente trabalho teve como objetivo estudar pela biomicroscopia ultra-sônica as alterações do segmento anterior em pacientes com síndrome de Sturge-Weber associada ou não a glaucoma.

\section{MÉTODOS}

Oito pacientes (onze olhos) portadores de síndrome de Sturge-Weber (dois olhos sem glaucoma e nove com glaucoma) foram submetidos à UBM. Em todos os pacientes, realizou-se, inicialmente, exame oftalmológico completo no qual foram incluídas: a anamnese (indagando a história de convulsões e/ou de uso de drogas anticonvulsivantes), a medida da acuidade visual sob melhor correção (quando possível), a biomicroscopia, a tonometria de aplanação com o tonômetro de Goldmann, a gonioscopia com a lente de três espelhos de Goldmann e a biomicroscopia de fundo de olho utilizando essa mesma lente.

Em seis olhos de três pacientes (1,2 e 7), foram consideradas as medidas da pressão venosa episcleral (Pv) feitas por Calixto e cols ${ }^{(12)}$. Nos outros pacientes, não foi possível ainda a realização dessa medida.

A UBM foi realizada em todos os olhos com angioma episcleral com os pacientes em decúbito dorsal, utilizando-se o biomicroscópio ultra-sônico modelo 840 (Humphrey-Paradigm), segundo técnica descrita por Pavlin et al ${ }^{(14)}$, obtendo-se cortes 
radiais da esclera, do corpo ciliar e região da pars plana. No paciente 3 ( 5 anos de idade) o exame oftalmológico e a UBM foram feitos sob narcose, tendo-se utilizado para a medida da pressão intra-ocular (Po), o tonômetro de aplanação de Perkins.

\section{RESULTADOS}

Em razão do pequeno número de olhos estudados, os resultados serão apresentados na forma de relatos de casos, não tendo sido realizada análise estatística.

\section{RELATO DOS CASOS}

Paciente 1: 28 anos, sexo masculino, leucodérmico. Negava história de convulsões. Ao exame, apresentava hemangioma facial à direita; acuidade visual igual a 1, sem correção, em AO; pressão intra-ocular (Po) igual a $13 \mathrm{mmHg}$ no OD e $12 \mathrm{mmHg}$ no OE. Ao exame biomicroscópico, apresentava formação plexiforme vascular vinhosa perilímbica em quase $360^{\circ}$ no OD poupando apenas a região de 3 às 7 horas; à gonioscopia, apresentava em ambos os olhos (AO), seio camerular amplo com sangue no canal de Schlemm; à fundoscopia, escavação papilar de 0,4 a 0,5 em AO e ausência de angioma de coróide no OD. A medida da Pv mostrou-se elevada em AO. Os achados da UBM no OD foram: imagem triangular intra-escleral de média ecogenicidade no quadrante inferior compatível com a suspeita diagnóstica de vaso intra-escleral dilatado (Figura 3a); imagem arredondada de baixa a média ecogenicidade no interior do corpo ciliar também no quadrante inferior, compatível com a suspeita diagnóstica de vaso intraciliar dilatado ou angioma do corpo ciliar (Figura 3b).

Paciente 2: 37 anos, sexo feminino, leucodérmica. Negava história de convulsões. Ao exame, apresentava hemangioma facial à esquerda; acuidade visual, sob correção, igual a 1,0 no olho direito (OD) e 0,4 no olho esquerdo (OE); Po igual a $12 \mathrm{mmHg}$ em AO; à biomicroscopia, não havia alterações no OD, porém o OE apresentava formação plexiforme vascular vinhosa na conjuntiva temporal. A gonioscopia do OE foi normal e a fundoscopia não mostrou angioma de coróide. A medida da Pv foi normal em AO. A UBM do OE mostrou apenas imagem de descolamento temporal da pars plana.

Paciente 3: 5 anos, sexo masculino, leucodérmico. História negativa de convulsão. Ao exame inicial aos 5 meses de vida, apresentava hemangioma facial à direita. Po igual a $5 \mathrm{mmHg}$ no OD e 2 mmHg no OE; à biomicroscopia, apresentava formação plexiforme vascular vinhosa perilímbica no OD; à gonioscopia, seio camerular amplo com sangue no canal de Schlemm do OD; a parede externa do seio camerular mostrava-se descoberta com franjado pectíneo na frente da faixa ciliar como prolongamento da periferia da íris em AO. À fundoscopia, apresentava escavação papilar de 0,2 a 0,3 no OD com a possível presença de angioma de coróide neste olho e escavação papilar de 0,1 a 0,2 no OE. Essa criança foi acompanhada durante quatro anos no Serviço tendo desenvolvido no OD glaucoma refratário ao tratamento clínico. Foi submetida a trabeculotomia no OD, com
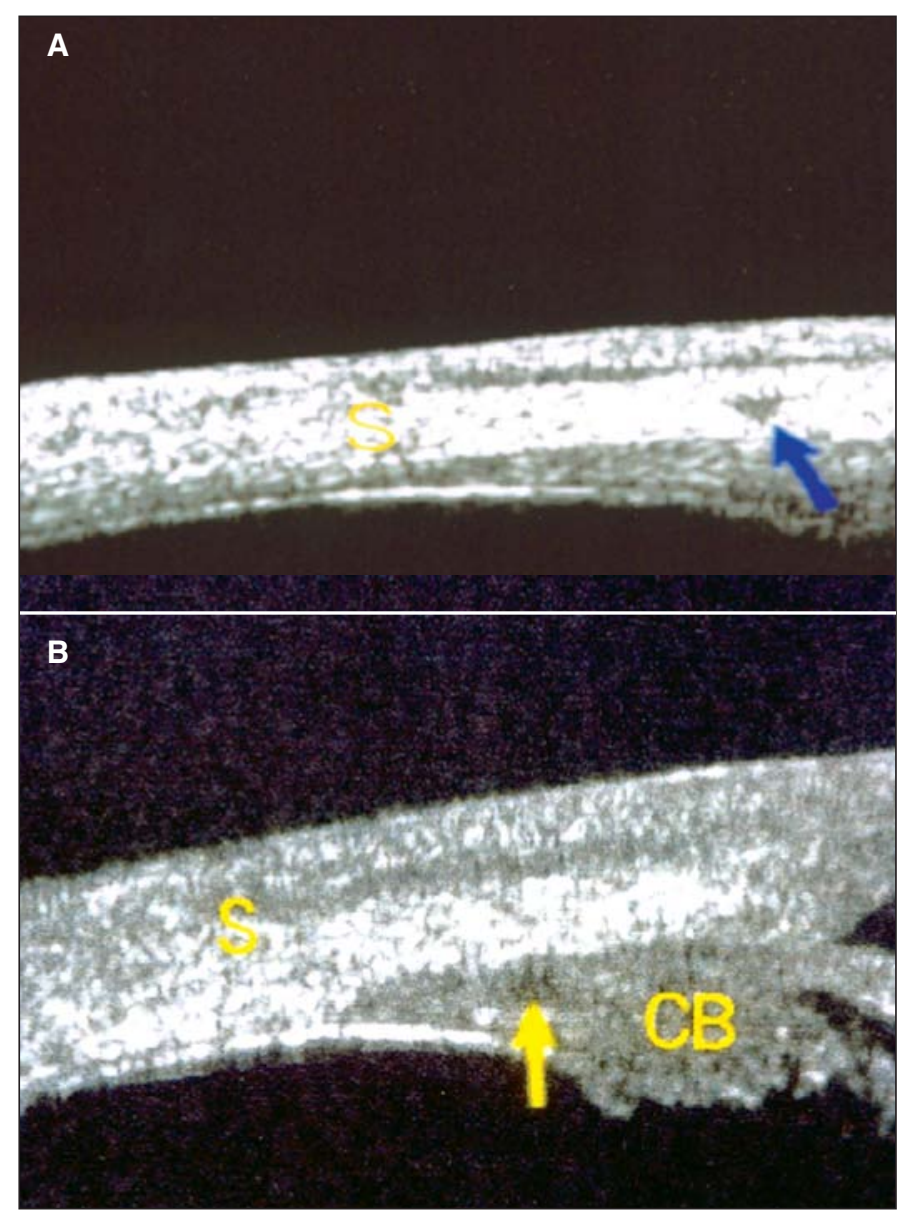

Figura 3 - Biomicrografia do paciente 1 ilustrando a presença de: A) uma imagem triangular de média ecogenicidade no interior da esclera compatível com a suspeita diagnóstica de vaso intra-escleral dilatado ou angioma (seta azul). B) uma imagem arredondada de baixa a moderada ecogenicidade no interior do corpo ciliar compatível com a suspeita diagnóstica de vaso intraciliar dilatado ou angioma do corpo ciliar

bom controle da Po sem medicação. Os achados da UBM realizada no OD, sem medicação, cerca de 20 dias após a cirurgia, foram: imagem triangular intra-escleral de média ecogenicidade no quadrante superior compatível com vaso intra-escleral dilatado (similar à fig. 3a); imagem arredondada de baixa a média ecogenicidade no interior do corpo ciliar também no quadrante superior, compatível com vaso intraciliar dilatado ou angioma do corpo ciliar ( similar à fig. 3b).

Paciente 4: 11 anos, sexo feminino, leucodérmica. História de convulsões na infância, sendo usuária de primidona $(250 \mathrm{mg}$ ao dia). Ao exame, apresentava hemangioma facial bilateral acometendo pálpebras e pescoço; acuidade visual igual a 0,35 no OD e 0,7 no OE, sob correção; Po igual a $36 \mathrm{mmHg}$ no OD e $32 \mathrm{mmHg}$ no $\mathrm{OE}$; à biomicroscopia, mostrava formação plexiforme vascular vinhosa no limbo nasal e temporal em AO; à gonioscopia, seio camerular do tipo intermediário com a raiz da íris aparentemente implantada ao nível do esporão escleral; à fundoscopia, mostrava papila atrófica com escavação de 0,9 no OD e papila rósea com escavação de 0,4 a 0,5 no $\mathrm{OE}$ e 


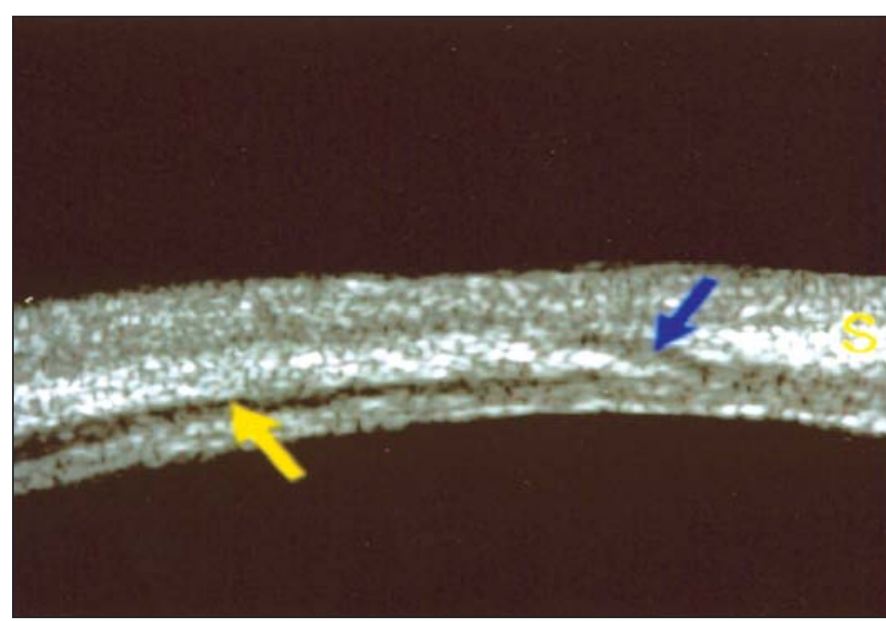

Figura 4 - Biomicrografia do paciente 4 ilustrando a presença de: imagem anecóica compatível com efusão supraciliar (seta amarela) e imagem no interior da esclera de baixa ecogenicidade de trajeto oblíquo compatível com a suspeita de vaso emissário (seta azul)

angioma de coróide em AO. Foi submetida a trabeculectomia com mitomicina $\mathrm{C}(0,3 \mathrm{mg} / \mathrm{ml}$ durante três minutos $)$, de urgência, em AO, usando-se no pré-operatório $250 \mathrm{ml}$ de manitol IV. Os achados da UBM realizada sob timolol, antes da cirurgia, foram em AO: imagem triangular intra-escleral de média ecogenicidade no quadrante superior compatível com vaso intraescleral dilatado (similar à fig. 3a); imagem arredondada de baixa a média ecogenicidade no interior do corpo ciliar nos quadrantes superior e temporal, compatível com vaso intraciliar dilatado ou angioma do corpo ciliar (similar à fig. 3b); efusão supraciliar (fig. 4, seta amarela) e descolamento da pars plana nos quadrantes superior e inferior no OD e no quadrante temporal no OE; imagem intra-escleral em fenda, de trajeto oblíquo, compatível com a possibilidade de vaso ou canal emissário (fig. 4, seta azul) no quadrante superior do OD. Quatorze meses após a cirurgia, a paciente apresentava Po igual a 6 mmHg sem medicação em AO. Repetiu-se a UBM em AO, encontrando-se achados similares aos do pré-operatório.

Paciente 5: 12 anos, sexo feminino, leucodérmica. Negava história de convulsão. Ao exame, apresentava hemangioma facial à esquerda; acuidade visual igual a 1, sem correção em AO; Po igual a 12 mmHg no OD e 24 mmHg no OE; à biomicroscopia, mostrava formação plexiforme vascular vinhosa justalímbica anular no $\mathrm{OE}$; à gonioscopia, mostrava no $\mathrm{OD}$, íris convexa para diante encobrindo parcialmente a faix ciliar na metade inferior e o esporão escleral na metade superior estando o canal de Schlemm exangue; no OE, parede externa do seio camerular apresentava-se descoberta com íris plana cuja raiz era prolongada por pectíneo em franjado na frente do esporão sendo que o canal de Schlemm mostrava-se temporariamente com sangue; à fundoscopia, observou-se escavação papilar de 0,1 a 0,2 no OD e de 0,3 a 0,4 no OE, evidenciando-se uma coloração vinhosa da coróide perimacular no $\mathrm{OE}$ (angioma de coróide). A paciente foi submetida a trabeculotomia em 10/02/94, sem sucesso e, posteriormente, a trabeculectomia em 13/07/95 com uso pré-operatório de $250 \mathrm{ml}$ de manitol intravenoso (IV), com sucesso parcial, estando atualmente em uso da associação de dorzolamida e timolol, uma gota duas vezes ao dia. Os achados da UBM no $\mathrm{OE}$, realizada cerca de cinco anos após a última cirurgia e sob dorzolamida e timolol, foram: imagem triangular intra-escleral de média ecogenicidade no quadrante inferior compatível com vaso intra-escleral dilatado (similar à fig. 3a); imagem arredondada de baixa a média ecogenicidade no interior do corpo ciliar no quadrante nasal, compatível com vaso intraciliar dilatado ou angioma do corpo ciliar (similar à fig. 3b) e efusão supraciliar (similar à fig. 4, seta amarela) nesse mesmo quadrante.

Paciente 6: 13 anos, sexo feminino, leucodérmica. Apresentava história de convulsões. Ao exame, apresentava angioma facial bilateral; acuidade visual igual a 0,5 sob correção em AO; Po igual a 6 mmHg em AO; à biomicroscopia, mostrava formação plexiforme vascular vinhosa perilímbica em AO; à gonioscopia, a parede externa do seio camerular apresentava-se descoberta e o canal de Schlemm exangue na metade nasal; restos pectíneos pouco desenvolvidos na frente de faixa ciliar com íris levemente côncava no OD e resíduos pectíneos aparentemente inseridos no esporão escleral no $\mathrm{OE}$. À oftalmoscopia, apresentava angioma de coróide em AO. Essa paciente foi inicialmente examinada aos seis meses de vida e desenvolveu o glaucoma após dois anos de seguimento, sendo tratada clinicamente por mais dois anos. Após este período, como a Po não se encontrava sob controle adequado, ela foi submetida à trabeculotomia aos cinco anos de idade, usando-se $250 \mathrm{ml}$ de manitol IV no pré-operatório, com bom controle da Po de AO, sem medicação. Os achados da UBM, realizada sete anos após a cirurgia e sem medicação em AO, foram: imagem triangular intra-escleral de média ecogenicidade no quadrante superior compatível com vaso intra-escleral dilatado (similar à fig. 3a); imagem arredondada de baixa a média ecogenicidade no interior do corpo ciliar também no quadrante superior, compatível com vaso intraciliar dilatado ou angioma do corpo ciliar ( similar à fig. 3b) e efusão supraciliar (similar à fig. 4, seta amarela) também nesse mesmo quadrante.

Paciente 7: 42 anos, sexo feminino, leucodérmica. Negava história de convulsões. Ao exame, apresentava angioma facial à direita; acuidade visual, sob correção, igual a 0,8 no OD e 1,0 no OE; Po igual a $24 \mathrm{mmHg}$ sob medicação no OD e $12 \mathrm{mmHg}$ sem medicação no $\mathrm{OE}$; à biomicroscopia, apresentava formação plexiforme vascular vinhosa inferonasal no OD e o OE normal; gonioscopia normal em AO; à oftalmoscopia, apresentava papila rósea com escavação de 0,2 e angioma coroidiano no OD. A medida da Pv mostrou-se elevada em AO. Foi realizada -no OD há 20 anos, usando-se $250 \mathrm{ml}$ de manitol IV no pré-operatório, com bom controle da Po sem medicação. Os achados da UBM, realizada no OD 19 anos após a cirurgia, sem medicação, foram: imagem triangular intra-escleral de média ecogenicidade no quadrante nasal compatível com vaso intra-escleral dilatado (similar à fig. 3a); imagem arredondada de baixa a média ecogenicidade no interior do corpo ciliar também no quadrante nasal, compatível com vaso intraciliar dilatado ou angioma do corpo ciliar (similar à fig. 3b) e efusão supraciliar (similar à fig. 4, seta amarela) no quadrante temporal.

Paciente 8: 26 anos, sexo feminino, leucodérmica. História 
de convulsão negativa. Ao exame inicial, realizado aos 13 anos de idade, apresentava angioma facial bilateral; acuidade visual igual a 1,0 sem correção, em AO; Po igual a $23 \mathrm{mmHg}$ no OD e $18 \mathrm{mmHg}$ no $\mathrm{OE}$, sem medicação; à biomicroscopia, apresentava formação plexiforme vascular vinhosa perilímbica em AO; à gonioscopia, seio camerular amplo com canal de Schlemm exangue no OD, porém com sangue no OE; à oftalmoscopia, apresentava papilas com escavações assimétricas $(0,6$ a 0,7 no $\mathrm{OD}$ e 0,5 no $\mathrm{OE}$ ) e angioma de coróide em AO. A paciente desenvolveu glaucoma bilateral e foi submetida a trabeculectomia em AO (OD em 1989 e OE em 1990) com o uso préoperatório de $250 \mathrm{ml}$ de manitol IV. Atualmente, a Po encontrase sob controle (17 $\mathrm{mmHg}$ ) em AO sob levobunolol, uma gota duas vezes ao dia. Os achados da UBM, realizada em AO, respectivamente 12 e 11 anos após a cirurgia e sob levobunolol, foram: imagem triangular intra-escleral de média ecogenicidade nos quadrantes superior e inferior no OD e no quadrante inferior no OE compatível com vaso intra-escleral dilatado (similar à fig. 3a); imagem arredondada de baixa a média ecogenicidade no interior do corpo ciliar nos quadrantes nasal e inferior no OD e nos quadrantes superior e inferior no $\mathrm{OE}$, compatível com vaso intraciliar dilatado ou angioma do corpo ciliar (similar à fig. 3b) e efusão supraciliar (similar à fig. 4, seta amarela) no quadrante temporal em AO.

A tabela 1 resume os dados demográficos, os achados clínicos e da UBM dos oito pacientes estudados.

\section{DISCUSSÃO}

O glaucoma na síndrome de Sturge-Weber tem sido objeto de várias publicações, sendo arroladas como causas do seu aparecimento, anomalias de desenvolvimento ou alterações degenerativas do seio camerular e/ou elevação da pressão venosa episcleral, sendo esta última a causa mais provável ${ }^{(1-3,5,8-13)}$. Neste trabalho, foram estudados pela UBM oito pacientes, todos com angioma episcleral, sendo nove olhos portadores de glaucoma ipsilateral aos angiomas episclerais e dois olhos sem glaucoma. Os dois olhos com angioma episcleral sem glaucoma não apresentavam angioma de coróide. Entretanto, oito $(88,9 \%)$ dos nove olhos glaucomatosos, apresentavam angioma de coróide, corroborando com os achados de outros autores $^{(5)}$.

Paradoxalmente, o achado mais importante da UBM foi a presença de efusão supraciliar apenas nos olhos com glaucoma (seis dos nove olhos glaucomatosos, metade deles em uso de medicação tópica antiglaucomatosa). A paciente 3 tinha glaucoma mas não efusão supraciliar. É possível que a paciente 3, no qual a UBM mostrou imagens compatíveis com vaso intraescleral dilatado e vaso ciliar dilatado ou angioma do corpo ciliar e cuja idade, na época da realização do exame, era de 5 anos, também apresentasse efusão supraciliar não diagnosticada pelas dificuldades inerentes à realização do exame (feito sob narcose e sem possibilidade de movimento dos olhos).

$\mathrm{O}$ achado de efusão supraciliar à UBM nos olhos portadores de glaucoma, seja no pré ou no pós-operatório, vem reforçar a conduta de utilizar-se um osmoterápico ( $250 \mathrm{ml}$ de manitol IV) no pré-operatório da cirurgia antiglaucomatosa de portadores da síndrome de Sturge-Weber, conduta esta que já vem sendo adotada há alguns anos no nosso Serviço, mesmo antes da detecção deste achado pela UBM. Esta droga evita ou atenua o aparecimento rápido de efusão coroidiana (após a paracentese do globo ocular) causada pela imediata transudação do fluido do espaço intravascular para o extravascular em conseqüência da Pv (episcleral e coroidiana) elevada. A hemorragia coroidiana expulsiva, complicação muito grave e temida, também pode ocorrer imediatamente após a paracentese do globo ocular nos olhos que apresentam angioma de coróide, estando este presente em $88 \%$ dos pacientes que desenvolvem glauco$\mathrm{ma}^{(6,9)}$. Nos olhos portadores de síndrome de Sturge-Weber associada a glaucoma é freqüente ocorrer hipertensão vítrea com perda de vítreo pelo orifício da trabeculectomia, aparentemente inexplicável, mesmo em cirurgia em que não ocorre nenhuma manobra intempestiva justificadora desta complicação.

Estudo prospectivo mostrou os resultados do emprego de latanoprost a $0,005 \%$ uma vez ao dia em seis olhos com glaucoma associado à síndrome de Sturge-Weber com Po não controlada com duas outras drogas antiglaucomatosas ${ }^{(16)}$. A Po foi medida no "baseline" e após tratamento com latanoprost com duração de pelo menos um mês. Dois (28\%) dos seis olhos demonstraram uma redução média da Po de $8,8 \mathrm{mmHg}$.

\begin{tabular}{|c|c|c|c|c|c|c|c|c|c|c|c|}
\hline \multirow[t]{2}{*}{ Caso } & \multirow{2}{*}{$\begin{array}{l}\text { Idade } \\
\text { (anos) }\end{array}$} & \multicolumn{2}{|c|}{ Po } & \multicolumn{2}{|c|}{ Glaucoma } & \multicolumn{2}{|c|}{ Pv } & \multirow[b]{2}{*}{$\begin{array}{c}\text { FO } \\
\text { Angioma } \\
\text { de } \\
\text { coróide }\end{array}$} & \multicolumn{3}{|c|}{ UBM } \\
\hline & & $\overline{O D}$ & OE & OD & OE & $\overline{O D}$ & $\mathrm{OE}$ & & $\begin{array}{c}\text { Vaso } \\
\text { intraescleral } \\
\text { dilatado }\end{array}$ & $\begin{array}{c}\text { Vaso } \\
\text { intraciliar } \\
\text { dilatado }\end{array}$ & $\begin{array}{c}\text { Efusão } \\
\text { supraciliar }\end{array}$ \\
\hline 1 & 28 & 13 & 12 & Não & Não & $\uparrow$ & $\uparrow$ & Não & Sim & Sim & Não \\
\hline 2 & 37 & 12 & 12 & Não & Não & $\mathrm{N}$ & $\mathrm{N}$ & Não & Não & Não & Não \\
\hline 3 & 5 & 5 & 2 & Sim & Não & NM & NM & Sim & Sim & Sim & Não \\
\hline 4 & 11 & 36 & 32 & Sim & Sim & NM & NM & Sim & Sim & Sim & Sim \\
\hline 5 & 12 & 12 & 24 & Não & Sim & NM & NM & Sim & Sim & Sim & Sim \\
\hline 6 & 13 & 6 & 6 & Sim & Sim & NM & NM & Sim & Sim & Sim & Sim \\
\hline 7 & 42 & 24 & 12 & Sim & Não & $\uparrow$ & $\uparrow$ & Sim & Sim & Sim & Sim \\
\hline 8 & 17 & 17 & 17 & Sim & Sim & NM & NM & Sim & Sim & Sim & Sim \\
\hline
\end{tabular}


Esses dois olhos que responderam ao latanoprost tinham, segundo os autores, glaucoma juvenil enquanto que os quatro olhos que não responderam tinham glaucoma congênito. Os autores concluíram que, em pacientes selecionados, o latanoprost pode reduzir significativamente a Po em pacientes com glaucoma associado à síndrome de Sturge-Weber.

Os achados desses autores ${ }^{(16)}$ e a presença de efusão supraciliar na maioria dos olhos portadores de glaucoma associado à síndrome de Sturge-Weber encontrada neste estudo, faz supor estar indicado nesses olhos o emprego dos análogos das prostaglandinas (latanoprost, bimatoprost ou travoprost), cujo mecanismo de redução da Po ocorre através do aumento do fluxo úveo-escleral. Supõe-se que esse tipo de medicação poderia diminuir a efusão supraciliar com conseqüente redução da Po. Há, entretanto, necessidade de estudos adicionais para comprovar esta hipótese.

O outro achado importante da UBM no presente estudo foi a imagem compatível com vaso intra-escleral dilatado tanto em olhos glaucomatosos como em olhos não glaucomatosos com angioma episcleral. Neste estudo, em apenas três olhos com angioma episcleral (pacientes 1, 2 e 7) (dois olhos sem glaucoma e um com glaucoma), a Pv havia sido medida anteriormente $^{(12)}$. Por isto, não foi possível correlacionar os achados da UBM com este parâmetro. Entretanto, dos três achados da UBM (Tabela 1), dois estavam presentes (não havia a efusão supraciliar) em um olho não glaucomatoso (paciente 1) com Pv elevada e os três no olho glaucomatoso com Pv elevada (paciente 7). Por outro lado, no outro olho não glaucomatoso (paciente 2) em que a $\mathrm{Pv}$ foi normal, não foram observadas alterações à UBM. Calixto e cols. ${ }^{(12)}$ não encontraram explicação para o aumento da Pv nos dois olhos com angioma episcleral sem glaucoma.

Em concordância com o relato de Kranemann et al ${ }^{(15)}$ todos os olhos aqui estudados, glaucomatosos ou não, apresentavam seio camerular amplo, sem anomalias típicas do glaucoma congênito. Também concordando com aqueles autores ${ }^{(15)}$, os achados do presente trabalho dão suporte à hipótese de que o mecanismo do glaucoma associado à síndrome de SturgeWeber, na maioria dos olhos, pode ser atribuído principalmente ao aumento da Pv, pelos seguintes motivos: 1. à gonioscopia, todos os olhos glaucomatosos apresentaram seio camerular amplo, sem anomalias típicas do glaucoma congênito; 2 . a maioria dos olhos glaucomatosos apresentou efusão supraciliar, achado este ausente nos olhos não glaucomatosos com angioma episcleral; 3. a maioria dos olhos glaucomatosos apresentou angioma de coróide, achado este ausente nos dois olhos não glaucomatosos; 4. ausência de inflamação intraocular (que poderia causar efusão supraciliar) em todos os olhos; 5. presença, ao exame gonioscópico, de sangue no canal de Schlemm em quatro olhos glaucomatosos. Este achado é excepcionalmente raro em olho glaucomatoso; 6. presença de efusão supraciliar apesar da normalização cirúrgica da Po, seja através da trabeculotomia ou da trabeculectomia.

A trabeculectomia pode ser a melhor opção quando o seio camerular não apresenta anomalias típicas do glaucoma congênito porque ela se desvia de qualquer componente do glaucoma causado pela Pv elevada enquanto isto não ocorre com a trabeculotomia e a goniotomia.
Em concordância com os motivos supramencionados, Jorgensen e Guthoff ${ }^{(11)}$ e Calixto et al ${ }^{(12)}$ encontraram a Pv elevada respectivamente em todos os 11 olhos glaucomatosos e em todos os 16 olhos glaucomatosos por eles estudados.

A efusão supraciliar ou supracoroidiana provavelmente é uma manifestação crônica da $\mathrm{Pv}$ elevada que por sua vez acarreta uma elevação da pressão venosa coroidiana.

Outro achado importante deste estudo, foi a presença de imagem arredondada de baixa a média ecogenicidade no interior do corpo ciliar, compatível com vaso intraciliar dilatado ou angioma do corpo ciliar ${ }^{(13)} \mathrm{em}$ dois olhos não glaucomatosos e em oito dos nove olhos portadores de glaucoma associado à síndrome de Sturge-Weber. Dos oito olhos portadores de glaucoma associado à síndrome de Sturge-Weber, apenas dois (paciente 8), necessitaram de medicação adicional (levobunolol, uma gota duas vezes ao dia), após a cirurgia, para o controle da Po. Estes dois olhos foram os que justamente apresentaram maior número de imagens sugestivas de alterações à UBM, o que faz supor que possa existir correlação entre a quantidade de alterações encontradas à UBM e a refratariedade do glaucoma associado ou o uso de drogas hipotensoras em olhos com alterações vasculares congênitas ou a soma dos achados. Há, entretanto, necessidade de ser estudado maior número de pacientes para comprovar esses achados.

\section{CONCLUSÕES}

Na síndrome de Sturge-Weber, a UBM mostrou a presença de imagens sugestivas de vasos dilatados no interior da esclera e do corpo ciliar (possíveis angiomas) em olhos glaucomatosos e não glaucomatosos.

Paradoxalmente, diagnosticou-se efusão supraciliar em oito $(88,9 \%)$ dos nove olhos com glaucoma.

Estes achados de UBM combinados com o angioma coroidiano e o aumento da pressão venosa episcleral em percentual elevado de olhos com esta síndrome, podem contribuir para explicar a patogenia do glaucoma, bem como as complicações per e pós-operatórias que acompanham as cirurgias antiglaucomatosas nesses olhos.

\section{ABSTRACT}

Purpose: To describe the ultrasound biomicroscopy findings in patients with Sturge-Weber syndrome with or without glaucoma. Methods: Ultrasound biomicroscopy was performed on 11 eyes from 8 patients with Sturge-Weber syndrome with or without glaucoma. Results: Ultrasound biomicroscopy showed images suggestive of dilated intrascleral vessels in all eyes with or without glaucoma. Supraciliar effusion was found in $8(88.9 \%)$ of 9 eyes with glaucoma and in none of those without glaucoma. Sectorial pars plana detachment was present in one eye with and in two eyes without glaucoma. A round image with low to moderate echogenicity in the ciliary body was present in 8 (88.9\%) eyes with glaucoma and in 2 
eyes without glaucoma. This image is highly suggestive of a dilated vessel or angioma. Conclusions: UBM showed paradoxically supraciliary effusion in the great majority of the eyes with glaucoma (89\%), controlled by previous surgery in the Sturge-Weber syndrome. The images found in the anterior portion of sclera and in the ciliary body are highly suggestive of a scleral dilated vessel and an angioma, respectively, in eyes with or without glaucoma. These findings associated with increased episcleral venous pressure might contribute to explain not only the pathogenesis of the secondary glaucoma but also the peri- and postoperative complications eventually present during and after the antiglaucomatous surgeries in these eyes.

Keywords: Sturge-Weber syndrome; Angiomatosis; Glaucoma/ultrasonography; Microscopy; Sclera/ultrasonography; Ciliary body/ultrasonography

\section{REFERÊNCIAS}

1. Schirmer R. Ein fall von telangiecktasie. Albrecht Von Graefes Arch Klin Exp Ophthalmologie 1860;7:119-21.

2. Sturge WA. A case of partial epilepsy apparently due to a lesion of the vasomotor centers of the brain. Trans Clin Soc (London) 1879;12:162-7.
3. Weber FP. A note of the association of extensive hemangiomatous nevus of the skin with cerebral (meningeal) hemangioma with contra lateral hemiplegia. Proc R Soc Med 1929;22:431-2.

4. Duhamel E, Goetz G: A propos de l'angiomatose encéphalotrigeminée. Bull Soc Ophtalmol Fr 1954;6:541-3.

5. Fitzpatrick TB, Kitamura H, Kukita A, Zeller R. Ocular and dermal melanocytosis. Arch Ophthalmol 1956;56:830-2.

6. Alexander GL, Norman RM. The Sturge-Weber syndrome. Bristol: John Wright \& Sons; 1960.

7. Weiss DI. Dual origin of glaucoma in encefalotrigeminal haemangiomatosis. Trans Ophthalmol Soc UK 1973;93:477-93.

8. Phelps CD. The pathogenesis of glaucoma in Sturge-Weber syndrome. Ophthalmology 1978;85:276-86.

9. Christensen GR, Records RE. Glaucoma and expulsive hemorrhage mechanism in the Sturge-Weber syndrome. Ophthalmology 1979;86:1360-6.

10. Cibis GV, Tripathi RC, Tripathi BJ. Glaucoma in Sturge-Weber syndrome. Ophthalmology 1984;91:1061-71.

11. Jorgensen JS, Guthoff R. Sturge-Weber syndrome: glaucoma with elevated episcleral venous pressure. Klin Monatsbl Augenheilkd 1987;191:275-8.

12. Calixto N, Cronemberger S, Coscarelli G, Cardoso RD. Encephalotrigeminal angiomatosis. Measurements of episcleral venous pressure and choroidal thickness. In: Krieglstein GK. editor. Glaucoma Update V. Heidelberg: Kaden; 1995. p.316-20.

13. Weiss JS, Ritch R. Glaucoma in the phakomatoses. In: Ritch R, Bruce Shields M, Krupin T. The glaucomas. 2ed. St. Louis: Mosby; 1996. p.907-14.

14. Pavlin CJ, Foster F. Ultrasound biomicroscopy of the eye. New York: Springer-Verlag; 1995.

15. Kranemann CF, Pavlin CJ, Trope GE. Ultrasound biomicroscopy in SturgeWeber - Associated Glaucoma. Am J Ophthalmol 1998;125:119-21.

16. Yang CB, Freedman SF, Myers JS, Buckley EG, Herndon LW, Allingham RR. Use of latanoprost in the treatment of glaucoma associated with SturgeWeber syndrome. Am J Ophthalmol 1998;126:600-2.

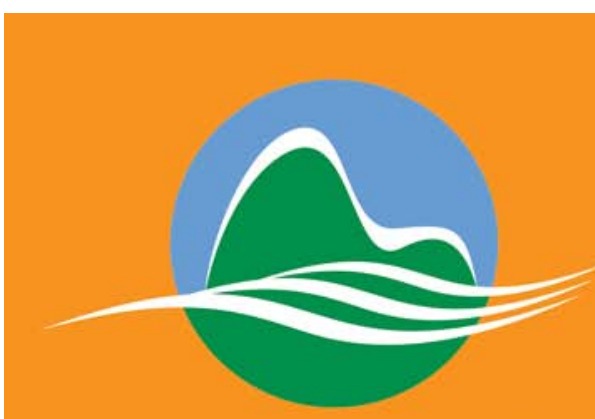

XVI CONGRESSO BRASILEIRO

DE PREVENÇÃO DA CEGUEIRA

E REABILITAÇÃO VISUAL

04 a 07 de setembro de 2004

Riocentro-Pavilhalo 5-Rio de Janeiro

PRESIDENTES

Riuitiro Yamane (RJ)

Yoshifumi Yamane (RJ)

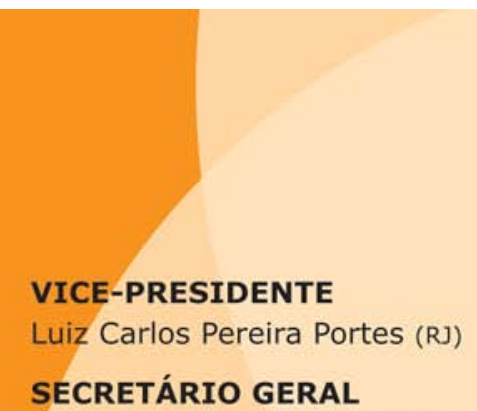

Renato Luiz Nahoum Curi (RJ)

1 ○ SECRETÁRIO

João Gabriel Costa (RJ)

TESOUREIRO

Antonio Luis Zangalli (RJ)

$1^{\circ}$ TESOUREIRO

Giovanni Colombini (RJ)

Estimativa do público:

$\mathbf{3 0 0 0}$ participantes
PROGRAMAÇÃO CIENTÍFICA Conferências / Simpósios / Cursos Temas Livres / Pôsteres / Vídeos Simpósios Satélites

\section{ATENÇÃO}

Data limite para envio de:

Vídeos: 17/06/04

\section{INFORMAÇÕES:}

Secretaria Executiva/Organização:

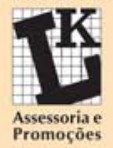

LK LTDA.

Tel.: (21) 3878-2512

Fax: (21) 3860-6064

E-mail: evento@lk.com.br

HP: www.cboprevcegueira04.com.br

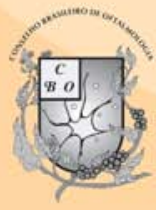

CBO

Fone: (11) 3266-4000

Fax: (11) 3171-0953

E-mail: eventos@cbo.com.br 